\title{
Processing of laser altimeter time of flight measurements to geodetic coordinates
}

Haifeng Xiao (1), Serena Annibali (2), Alexander Stark (2), Hauke Hussmann (2), Jürgen Oberst $(1,2)$

(1) Technical University of Berlin, Institute of Geodesy and Geoinformation Science, Berlin, Germany

(haifeng.xiao@campus.tu-berlin.de), (2) German Aerospace Center (DLR), Institute of Planetary Research, Berlin, Germany

Laser altimeters (LAs) are extensively used in planetary research due to their high ranging accuracy. The geolocation of LA time of flight (ToF) measurements is a key procedure towards the scientific analysis of the data and entails various aspects affecting its geodetic accuracy. We propose four progressively refined models to investigate the effects of different approximations: (1) Simultaneity Model (SM) which assumes all events within the measurement process to be simultaneous; (2) Spacecraft's Motion Model (SMM) that accounts for the spacecraft's motion during ToF; (3) Pointing Aberration Model (PAM) which takes a further step to take care of the pointing aberration induced by the relative velocity of the spacecraft with respect to the observer and (4) Special Relativity Model (SRM) that uses the Lorentz transformation to directly convert the coordinates in the spacecraft's frame to those on the target body.

These models are then applied to selected Mars Orbiter Laser Altimeter (MOLA) Precision Experiment Data Records (PEDR) (only orbital profiles, involving nadir pointing on a circular orbit) and Mercury Laser Altimeter (MLA) Reduced Data Records (RDR) profiles (orbital profiles and flyby profiles, both featuring large off-nadir angles on an eccentric orbit). Note that the released data records include both the raw ToF measurements and geolocated coordinates of Laser footprints (which appear to be computed using the SMM model). We compare results from the SMM model with those from the advanced PAM model, which includes the pointing aberration effect. While we obtain insignificant improvements of 4 to $5 \mathrm{~m}$ laterally and up to $\pm 3 \mathrm{~cm}$ radially for MOLA profiles, the difference is significant up to $150 \mathrm{~m}$ laterally and $\pm 25 \mathrm{~m}$ radially for the MLA orbital profiles and up to $100 \mathrm{~m}$ laterally and $-50 \mathrm{~m}$ radially for the MLA flyby profiles. 